\title{
Computing Sentiment Analysis through Aspect-based fuzzy Aggregations
}

\author{
Jesus Serrano-Guerrero and Jose A. Olivas and Francisco P. Romero \\ Department of Technologies and Information Systems, \\ University College of Computer Science, University of Castilla-La Mancha, \\ Paseo de la Universidad 4, 13071, Ciudad Real, Spain, \\ jesus.serrano@uclm.es, joseangel.olivas@uclm.es, franciscop.romero@uclm.es
}

\begin{abstract}
The expression of a general opinion on a product or service may sometimes be broken down into different sub-opinions on the different aspects which characterize such a product or service. Sometimes, the general opinion about a product does not have to be the average of the other sub-opinions, but other user-dependent factors can make the aggregation of the sub-opinions more complex.

This work studies some use cases with real opinions, in which fuzzy aggregation operators can help represent certain user behaviors, and hence, explain the overall assessment of an opinion with respect to partial opinions of aspects.
\end{abstract}

Keywords: Sentiment analysis, Aspects level, Fuzzy aggregation operators

\section{Introduction}

Retrieving information containing opinions towards different products is recently becoming a real need for many users on the Internet. Many important companies on Internet like Ebay ${ }^{1}$, allow expressing sentiments about their products or services, and even, about the aspects of those products or services. Nevertheless, when a user search for some information about a specific product, the only available mechanism is the selection of those opinions sharing the same overall rating.

Sometimes, the overall opinion of a product or service has nothing to do with the opinion of the subaspects that characterize that product or service as can be seen in the figure 1 . The overall score is 3 bubbles, while the rest of the aspects have scores higher than 3 .

\footnotetext{
${ }^{1}$ https://www.ebay.com
}

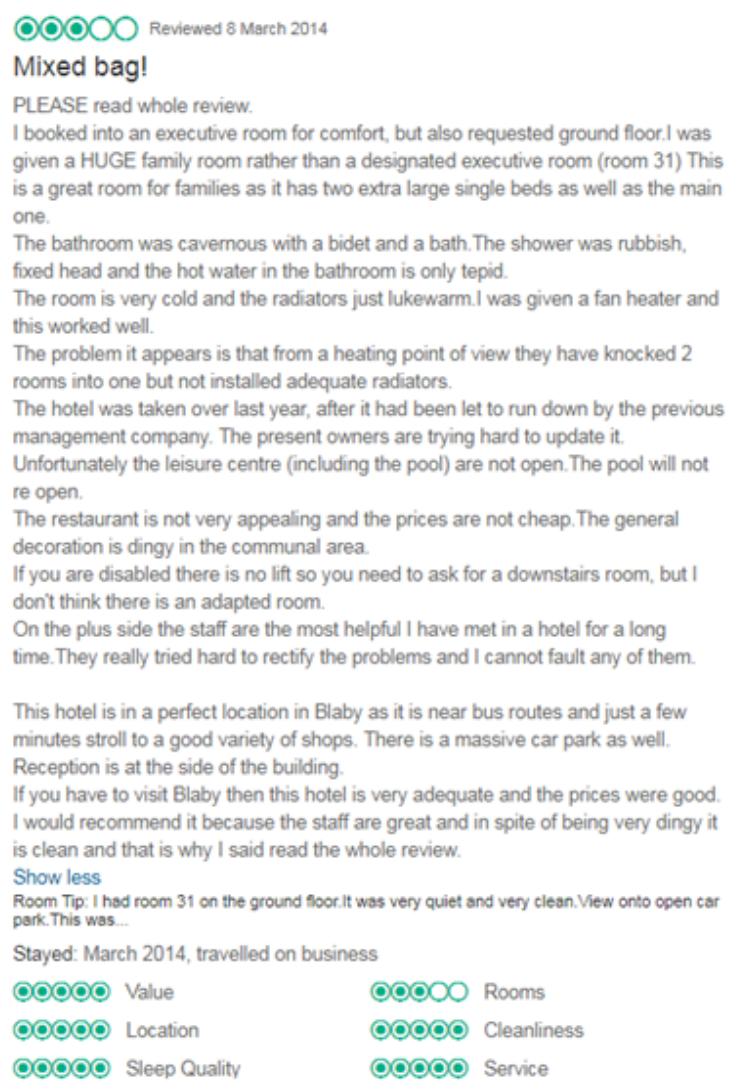

Figure 1: Opinion from Tripadvisor

This does not mean that the overall opinion is inconsistent, but simply that the average of the sub-opinions does not have to reflect the final opinion of the user. It is necessary to study aspects that may influence the overall assessment and propose other mechanisms to understand the overall scoring of an opinion. Therefore, the main contributions of this work are : (i) study of different behaviours of users when giving their opinion on a product, (ii) proposing various methods of aggregation of sub-opinions to obtain the global score of a product, and (iii) testing the proposed mechanisms through several examples. 
The remainder of the paper is organized as follows: the section 2 analyzes the most important articles on the topics treated here. The section 3 presents services by Google which will be used to test these proposals. The section 4 proposes several way to aggregate opinions, including some examples. Finally, the section 5 points out some conclusions and future works.

\section{State of the Art}

\subsection{Sentiment Analysis}

The interest in Sentiment analysis, also called Opinion Mining, has been growing over the last years. Its main purpose is to analyze people's sentiments, opinions, attitudes, emotions, etc., towards targets such as services, products, individuals, organizations, among others.

Opinion Mining is a field related to other areas like Artificial Intelligence (AI) or Natural Language Processing (NLP), among others. Among the main challenges that NLP has to cope with are the different levels of analysis. Depending on whether the target of study is a whole text or document, one or several sentences from the some source, or one or several aspects from different identified entities, it is necessary to face different kinds of subtasks. Three levels of analysis clearly determine the different subtasks of Opinion Mining: document level, sentence level and entity/aspect level.

The document level considers a document as a whole opinion. This level is closely related to the task called document-level sentiment classification $[34,13,6,9$, 17, 10]. However, when a document encloses several phrases dealing with different aspects or entities, it is necessary to focus on the sentence level. This one is related to subjectivity classification, a subarea which distinguishes phrases expressing factual information from phrases containing more subjective views $[20,2,4,3]$. And finally, the finest-grained level is the aspect level, which considers a target on which the user expresses an opinion in a positive or negative sense [25, 16, 29].

Sentiment Analysis can comprise many different subfields such as Sentiment Classification, Subjectivity Classification, Opinion Retrieval, among others [19, 23]. In particular, this work might be closely related to Opinion Summarization, which is also called Opinion Aggregation [30].

The problem treated here can be faced from two different points of view: single-document and multidocument summarization. Single-document summarization is based on analyzing internal aspects present within the analyzed document, for example, changes in the sentiment orientation throughout the document or links between the different entities/aspects found, and mainly showing those pieces of texts which better describe them. On the other hand, in multi-document summarization once aspects and entities have been detected, the system has to group and/or order the different sentences which express sentiments related to those entities or aspects. The final summary might be presented as a graphic or a text showing the main aspects/entities and quantifying the sentiment with regard to each one in some way, for example, aggregating intensities of sentiments or counting the number of positive or negative sentences [18, 15, 14, 7, 8, 24].

In Opinion Summarization [27], the aggregation process for the different elements of an opinion, could involve several steps as shown in figure 2 .

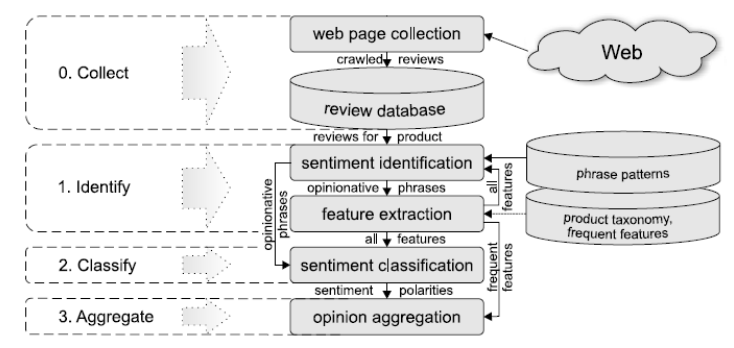

Figure 2: Architecture of product review aggregation $[27]$

To the best of our knowledge some papers can be found focused on a particular stage from the above figure but not especially only focused on the aggregation step.

Thus, there are some articles aggregating sentences to obtain the general sentiment for a document [5]; and some other papers trying to predict individual aspect ratings $[31,21]$. It is stated that "most methods aggregate sentiment by simply averaging or taking a majority vote" [22], i.e, basic approaches may be found and mainly only numerical not linguistic based.

For instance, in [28] an ontology-based framework is described, which ranks the main aspects of different products but, in this case, considering as a main factor the aggregation of the user preferences detected. In [1] an other similar approach can be found, which weighs the importance of the different aspects considering the levels of Conceptnet[12], and those weights are used to aggregate the sentiments about each aspect.

Zhang proposed several numerical approaches to aggregate the polarities from several aspects from different products like the mean or a weighted scheme depending on the importance of each aspect [35]. And for example, in [11] it is proposed an aggregation process taking into account a semantic score and a credibility score as main aspects to obtain the sentiment score towards some products. 


\subsection{Ordered Weighted Averaging (OWA) operator}

An Ordered Weighted Averaging (OWA) operator of dimension $n$ is defined as a mapping $F: R^{n} \rightarrow R$ which has an associated weighting vector $W=$ $\left[w_{1}, w_{2}, \ldots, w_{n}\right]$ in which $w_{j} \in[0,1]$ and $\sum_{j=1}^{n} w_{j}=1$ and where $F\left(a_{1}, a_{2}, \ldots, a_{n},\right)=\sum_{j=1}^{n} w_{j} b_{i}$, with $b_{j}$ being the $j$ th largest of the collection of the aggregated objects $a_{i}$.

One of the main aspects of the OWA operators is re-ordering step, each element $a_{i}$ is not associated with a particular weight $w_{i}$ but each weight $w_{i}$ is associated with a particular position $i$ of the ordered elements. The type of aggregation performed by an OWA operator depends upon the form of the weighting vector, therefore, by the selection of the appropriate vector $\mathrm{W}$, the OWA operators can model the max, min and arithmetic mean operators. Thus given the vectors $W=[1,0,0, \ldots, 0]$, $W=[0,0,0, \ldots, 1]$ and $W=[1 / n, 1 / n, \ldots, 1 / n]$, then $f\left(a_{1}, \ldots, a_{n}\right)=\operatorname{Max}\left(a_{i}\right), f\left(a_{1}, \ldots, a_{n}\right)=\operatorname{Min}\left(a_{i}\right)$ and $f\left(a_{1}, \ldots, a_{n}\right)=\frac{1}{n} \sum_{i=1}^{n} a_{i}$, respectively.

Two characterizing measures associated with the weighting vector $W$ of an OWA operator were introduced by Yager [32]. The first one is known as the measure of orness of the aggregation and is defined as

$$
\operatorname{orness}(W)=\frac{1}{n-1} \sum_{i=1}^{n}(n-i) w_{i}
$$

This measure characterizes the degree to which the aggregation is similar to an OR operation. On the other hand the other measure calculates the dispersion of the aggregation and is defined as

$$
\operatorname{disp}(W)=-\sum_{i=1}^{n} w_{i} \ln w_{i}
$$

On the contrary this measure assesses the degree to which $W$ takes into account all information in the aggregation.

Yager proposed the Regularly Increasing Monotonic (RIM) quantifiers as way of obtaining a weighting vector $W$ associated with an OWA aggregation [33]. Given a RIM quantifier $Q$, then we can compute an OWA weighting vector $W$ associated with $Q$ such that for $j \in\{1, \ldots, n\}$ :

$$
w_{j}=Q\left(\frac{j}{n}\right)-Q\left(\frac{j-1}{n}\right)
$$

Therefore, the linguistic expression of the quantifier can help us calculate the weighting vector $W$ and to include any meaning in the aggregation.

\subsubsection{WOWA operator}

Torra proposed the Weighted OWA (WOWA), which combines the OWA operator and the weighted mean together. On the one hand, as in the weighted mean, some weights measure the importance of an information source with independence of the value that the source has captured. On the other hand, as in the OWA, some other weights measure the importance of a value (in relation to other values) with independence of the information source. It is a generalization of both the weighted mean and the OWA operator allowing having the parameters of both operators into a single one. This operator was introduced by Torra in order to consider situations where both the importance of information source and the importance of values had to be taken into account [26]:

$$
\begin{gathered}
W_{P}^{w}: R^{n} \rightarrow R \\
W_{P}^{w}\left(x_{1}, x_{2}, x_{3}, \ldots, x_{n}\right)=\Sigma_{i=1}^{n} \mu_{i} x_{\partial}(i)
\end{gathered}
$$

Where $\partial$ is the permutation of $\{1,2,3, . ., n\}$ such that $X_{\alpha(1)} \geq X_{\alpha(2)} \ldots \geq X_{\alpha(n)}$ and weights $\mu_{i}$ are defined as:

$$
\mu_{i}=F\left(\Sigma_{j=1}^{i} P_{\partial(j)}\right)-F\left(\Sigma_{j=1}^{i-1} P_{\partial(j)}\right)
$$

Where $\mathrm{F}$ is a non-decreasing function that interpolates the points $\left(\frac{i}{n}, \sum_{j=1}^{i} W_{j}\right)$ together with the point $(0,0)$.

\section{Google Cloud Natural Language $\mathrm{API}^{2}$}

Google provides several services related to Natural Language Processing, among them, some related to Sentiment Analysis. It is possible to analyze the polarity for a whole opinion as well as for individual sentences or entities from that opinion. The different parameters that this API can compute are:

- Magnitude: How emotional an opinion is. If an opinion is very emotional but the score is close to zero means that the are mixed feelings.

- Salience: Importance or relevance of the entity to the whole opinion.

- Score: It expresses the polarity of an entity, sentence or whole opinion. It can be positive or negative.

\footnotetext{
${ }^{2}$ https://cloud.google.com/natural-language/
} 


\section{Proposal}

Two approaches are proposed to aggregate opinions based on their aspects, a simpler one based only on the stars or bubbles assigned to each product or service, and a more complex one based on the importance of each aspect, as well as the score of every individual aspect characterizing each product or service.

\subsection{Aggregation according to bubbles}

In this case it is proposed the use of the OWA operator to model the aggregation of stars or bubbles. The weights vector $\mathrm{W}$ will allow to model different behaviours of the users according to their mood, while the values A will represent the opinion expressed for each aspect.

\section{User modeling}

For these examples we will consider the opinions collected from the web Tripadvisor ${ }^{3}$ where the user can express the general opinion about his stay (look at the top of the pictures) and the specific opinion measured using bubbles about different aspects related to his stay: Value, Location, Sleep Quality, Room, Cleanliness and Service.

The first thing necessary to do, is to anlayse the different types of users can be found:

\section{Negative}

A bad-tempered user may draw a not-so-positive conclusion about a particular product or service by taking only one aspect into account, even if the assessment of the rest of the aspects is not so negative. Thus, in this case, the weight vector of the OWA operator could be used, simulating a minimum value. Let's look at the following example:

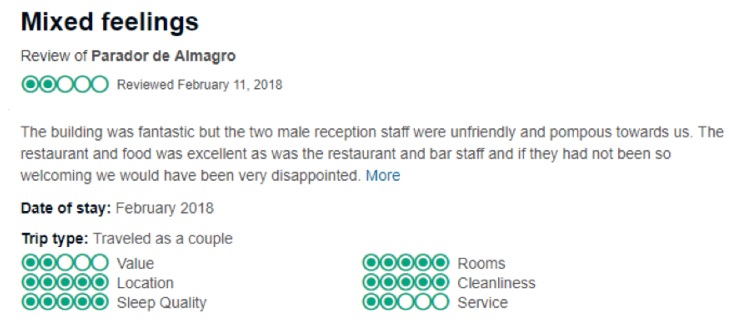

Figure 3: Opinion from TripAdvisor

The user, in spite of having a good opinion on the different aspects in general lines, expresses his disappointment about the staff for an incident. For that reason, he decides to give the

\footnotetext{
${ }^{3}$ https://developer-tripadvisor.com/contentapi/business-content/subratings/
}

minimum score for the whole opinion (see the bubbles on the top). In this case, modeling the feeling of the user through an arithmetic mean would not make sense because the value would be 4 , while if we use an OWA operator that favors the minimum value through its weights, for example, $W=\{0.5,0.2,0.1,0.1,0.1,0.0 .0\}$ where $a_{\sigma}=\{2,2,5,5,5,5,5\}$, it would be 2.9 , which is much closer to representing the behavior of the user.

\section{Balanced}

Normally, a user who objectively evaluates a product or service tends to calculate the average of the opinions of the aspects in order to give an objective view of it. For this reason, the use of the arithmetic mean can be proposed or the use of an OWA vector whose weights vector make more important the central values of the opinions.

Let's look at the following example:

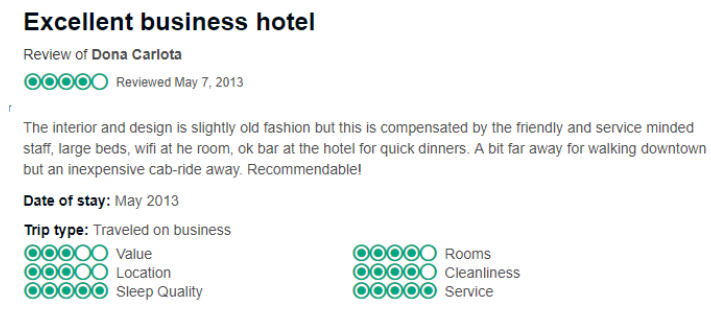

Figure 4: Opinion from TripAdvisor

This opinion clearly expresses an arithmetic mean of the values of the aspects. The overall value is 4 bubbles which would be the average of the bubbles for each aspect $(3,3,5,4,4,5)$.

If this user's opinion were to be modeled through an OWA operator, greater weight would be given to the central values, for example through the vector $W=\{0.0,0.2,0.3,0.3,0.2,0.0\}$ being $A_{\sigma}=$ $\{3,3,4,4,5,5\}$. The final result of the aggregation would also be 4 .

\section{Positive}

A user can express a very positive opinion of a certain product or service taking into account only some of its specific aspects, because it covers their needs, although the assessment of the rest of aspects is not so positive. Thus, in this case, the weight vector of the OWA operator could be used, simulating a maximum value.

Let's look at the following example:

In this case, the user has more aspects of 4 points than of 5 , however, the final rating is 5 . In this case, the arithmetic mean is 4.3 , while if an OWA operator were used taking into account 


\section{Excellent Parador}

Review of Parador de Almagro

OOOOO Reviewed October 10, 2017

Excellent Parador within easy walking distance to all the main attractions of Almagro. The Nationa Theatre Museum is well worth a visit. The staff and service provided was first class. The restaurant menu was extremely good. More

Date of stay: October 2017

Trip type: Traveled as a couple

OOOOO Value

OOOOO Location

OOOOO Rooms OOOOO Cleanliness OOOOO service

Figure 5: Opinion from TripAdvisor

the weights $W=\{0.0,0.0,0.1,0.1,0.3,0.5\}$ being $a_{\sigma}=\{4,4,4,4,5,5\}$, the final result would be 4.8 which would be closer to the result expressed by the user.

\subsection{Aggregation according to textual opinions}

The use of the stars or bubbles used in an opinion is slightly limited because it does not allow to reach what the user really wanted to express, for this reason, such information can be complemented with the use of textual information expressed. From this information certain conclusions can be drawn, such as what the most important aspect is for a user when expressing his final assessment of a product. Not all aspects necessarily have to be of equal importance to a user, and therefore, do not affect the final assessment of the opinion in the same way.

Therefore, a series of phases are proposed to calculate the overall sentiment of an opinion on a given product or service. These phases are related to the proposed ones in figure 2 :

1. Detection of opinionated entities: It is necessary to use NLP techniques such as ER (Entity Recognition) to detect the most representative entities in each opinion. In this case the Google Cloud Natural Language service mentioned above has been used. In addition, the sentences where each entity is, are also detected.

2. Aspects classification: It is necessary to associated each entity with each of the aspects that characterize any product or service.

In this case, more than 100,000 opinions collected from Tripadvisor have been processed, and the 2,000 most repeated words have been selected. Subsequently they have been classified manually, among the 6 aspects that Tripadvisor uses, generating 6 bags of words. In order to detect whether or not an entity belongs to a specific aspect, each bag of words is analysed. If the word is not found, the it is searched in Conceptnet ${ }^{4}$ evaluating the distance between the searched entity and the words of each bag of words. The aspect that has the greatest number of words with the lowest distance from the entity treated, will be the aspect in which it will be classified. It is also possible not classify a word for not being close enough to a specif bag of words.

3. Detection of sentiment: It is necessary to know the associated sentiment of each each sentence to know. Each sentence is talking about a specific aspect depending on the entities contained. For this, the magnitude score provided by the Google Cloud Natural Language service is used.

4. Importance of each entity: Not all aspects can have the same importance for a user, therefore, it is necessary to know the user or interpret from the textual opinion which are the most determining aspects to make the final decision on the assessment of a product or service. In this case, given Tripadvisor's users are not known, we propose the use of the magnitude salience of the Google Cloud Natural Language service, as a measure of the importance of each entity within each aspect. The average of all entities in each aspect will give the relative importance of each aspect.

5. Aggregation of aspects according to the importance of each aspect:

Once calculated the importance of each aspect, and the sentiment of each subaspect mainly through the magnitudes score and salience of Google Cloud Natural Language, the system can add all the values, but this time taking into account the relative importance of each aspect with respect to the final opinion. To do this, we propose aggregation through the WOWA operator introduced by Torra. In this case, the importance of each aspect will be considered as the importance vector of the information source $P=\left\{\right.$ salience $_{1}$, salience $_{2}$, salience $_{3}$, salience $_{4}$, salience $_{5}$, salience $\left._{6}\right\}$, while to model the weight vector $W=\{1 / 6,1 / 6,1 / 6,1 / 6,1 / 6,1 / 6\}$ an balanced distribution might be used. The input values $A=$ $\left\{\right.$ score $_{1}$, score $_{2}$, score $_{3}$, score $_{4}$, score $_{5}$, score $\left._{6}\right\}$ will represent the score of each aspect.

Given the following opinion,

it has been processed according to the previous steps and all values within the range $[0,1]$ have been normalized. The first sentence refers to the "Value" aspect whose salience is 0.432 and the score is 0.3 once

\footnotetext{
${ }^{4} \mathrm{http}: / /$ conceptnet.io
} 


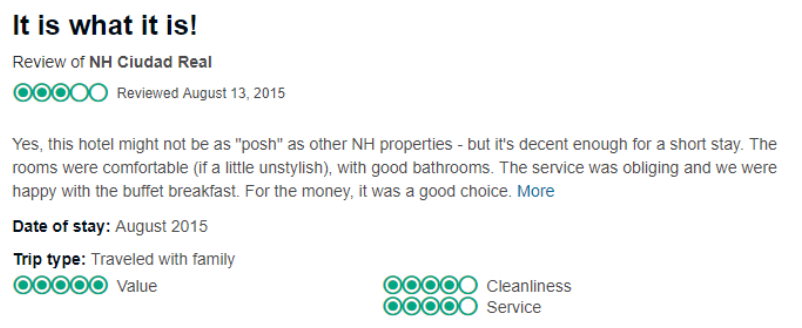

Figure 6: Opinion from TripAdvisor

normalized. While the second sentence refers to the "Rooms" aspect and its salience is lower, 0.02 and its score 0.94. Similar values are obtained with the third sentence refering to the aspect "Service", with salience 0.015 and score 0.94. The last phrase has not been associated with any aspect because it is too generic.

In this example only 3 aspects are going to be added because the user has not given information about the rest, which does not mean that he scored them as zero. For the calculation of the operator WOWA and OWA has been used the library developed by Torra ${ }^{5}$.

Considering the vector $W=\{1 / 3,1 / 3,1 / 3\}$, and using the WOWA operator, the final value of the aggregation would be 0.343 , while only using the OWA operator the value 0.72 . Obviously, the value is closer to what the user thinks, because Google Cloud has been able to assess in this case, which the most important aspect for the user is. Therefore, this proposal success is based on a good detection of the most important entities within the text thanks to NLP techniques.

\section{Conclusions and future works}

Two approaches have been presented to model the aggregation process of different aspects from user opinions through different fuzzy operators. These approaches allow modelling aspects such as the character of a user or the relative importance of each aspect for each user, which allows explaining and personalising the final score of a product or service depending on each user.

It is necessary to study in depth different aspects such as the different profiles that different users may represent, as well as to analyse the amount of information needed to apply these techniques, especially due to the recurrent problem of short texts.

New future work comes up from these approaches, such as the search for opinions based on specific aspects and their scores, or the recommendation of products or services based on their opinions but in a more guided

\footnotetext{
${ }^{5}$ http://www.mdai.cat/ifao/wowa.php
}

way, not only taking into account the general opinion but focusing on certain aspects.

\section{Acknowledgement}

This work has been partially supported by FEDER and the State Research Agency (AEI) of the Spanish Ministry of Economy and Competition under grant TIN2016-76843-C4-2-R (AEI/FEDER, UE).

\section{References}

[1] B. Agarwal, N. Mittal, Sentiment Analysis Using ConceptNet Ontology and Context Information, in: N. Mittal (Ed.), Prominent Feature Extraction for Sentiment Analysis, Springer International Publishing, 2016, pp. 63-75.

[2] O. Appel, F. Chiclana, J. Carter, H. Fujita, A hybrid approach to the sentiment analysis problem at the sentence level, Knowledge-Based Systems 108 (C) (2016) 110-124.

[3] O. Appel, F. Chiclana, J. Carter, H. Fujita, A Consensus Approach to the Sentiment Analysis Problem Driven by Support-Based IOWA Majority, International Journal of Intelligent Systems 32 (9) (2017) 947-965.

[4] O. Appel, F. Chiclana, J. Carter, H. Fujita, Crossratio uninorms as an effective aggregation mechanism in sentiment analysis, Knowledge-Based Systems 124 (C) (2017) 16-22.

[5] M. E. Basiri, A. R. Naghsh-Nilchi, N. GhasemAghaee, Sentiment prediction based on dempstershafer theory of evidence, Mathematical Problems in Engineering 2014 (2014) 1-13.

[6] A. Duric, F. Song, Feature selection for sentiment analysis based on content and syntax models, Decision Support Systems 53 (4) (2012) 704-711.

[7] K. Ganesan, C. Zhai, J. Han, Opinosis: a graphbased approach to abstractive summarization of highly redundant opinions, in: Proceedings of the 23rd International Conference on Computational Linguistics (COLING' '10), 2010, pp. 340-348.

[8] K. Ganesan, C. Zhai, E. Viegas, Micropinion generation: An Unsupervised Approach to Generating Ultra-Concise Summaries of Opinions, in: Proceedings of the 21st international conference on World Wide Web - WWW '12, ACM Press, 2012, pp. $869-878$.

[9] Y. He, D. Zhou, Self-training from labeled features for sentiment analysis, Information Processing and Management 47 (4) (2011) 606-616. 
[10] G. Li, F. Liu, Application of a clustering method on sentiment analysis, Journal of Information Science 38 (2) (2012) 127-139.

[11] Y. M. Li, T. Y. Li, Deriving market intelligence from microblogs, Decision Support Systems 55 (1) (2013) 206-217.

[12] H. Liu, P. Singh, ConceptNet - A Practical Commonsense Reasoning Tool-Kit, BT Technology Journal 22 (2004) 211-226.

[13] R. Moraes, F. Valiati, J, W. P. Gavião Neto, Document-level sentiment classification: An empirical comparison between SVM and ANN, Expert Systems with Applications 40 (2) (2013) 621633.

[14] H. Nishikawa, T. Hasegawa, Y. Matsuo, G. Kikui, Opinion summarization with integer linear programming formulation for sentence extraction and ordering, in: Proceedings of the 23rd International Conference on Computational Linguistics (COLING' '10), 2010, pp. 910-918.

[15] H. Nishikawa, T. Hasegawa, Y. Matsuo, G. Kikui, Optimizing informativeness and readability for sentiment summarization, in: Proceedings of the Association for Computational Linguistics (ACL'10), 2010, pp. 325-330.

[16] B. A. Ojokoh, O. Kayode, A feature-opinion extraction approach to opinion mining, Journal of Web Engineering 11 (1) (2012) 51-63.

[17] G. Paltoglou, M. Thelwall, A study of information retrieval weighting schemes for sentiment analysis, in: Proceedings of the 48th Annual Meeting of the Association for Computational Linguistics (ACL '10), 2010, pp. 1386-1395.

[18] K.-M. Park, H. Park, H.-G. Kim, H. Ko, Review summarization based on linguistic knowledge, in: H. Yu, G. Yu, W. Hsu, Y.-S. Moon, R. Unland, J. Yoo (Eds.), Proceedings of the 17th international conference on Database Systems for Advanced Applications (DASFAA'12), Vol. 7240 of Lecture Notes in Computer Science, Springer Berlin Heidelberg, Berlin, Heidelberg, 2012, pp. 105-114.

[19] K. Ravi, V. Ravi, A survey on opinion mining and sentiment analysis: Tasks, approaches and applications, Knowledge-Based Systems 89 (2015) 1446.

[20] R. Remus, C. Hänig, Towards well-grounded phrase-level polarity analysis, in: Proceedings of the 12th international conference on Computational linguistics and intelligent text processing (CICLing'11), 2011, pp. 380-392.
[21] C. Sauper, R. Barzilay, Automatic aggregation by joint modeling of aspects and values, Journal of Artificial Intelligence Research 46 (2013) 89-127.

[22] K. Schouten, F. Frasincar, Survey on AspectLevel Sentiment Analysis, IEEE Transactions on Knowledge and Data Engineering 28 (3) (2016) 813-830.

[23] J. Serrano-Guerrero, J. A. Olivas, F. P. Romero, E. Herrera-Viedma, Sentiment analysis: A review and comparative analysis of web services, Information Sciences 311 (1) (2015) 18-38.

[24] S. Tata, B. Di Eugenio, Generating Fine-Grained Reviews of Songs from Album Reviews, in: Proceedings of the 48th Annual Meeting of the Association for Computational Linguistics, Association for Computational Linguistics, Uppsala, Sweden, 2010, pp. 1376-1385.

[25] T. T. Thet, J.-C. Na, C. S. G. Khoo, Aspectbased sentiment analysis of movie reviews on discussion boards, Journal of Information Science 36 (6) (2010) 823-848.

[26] V. Torra, Weighted OWA operators for synthesis of information, in: Proceedings of IEEE 5th International Fuzzy Systems, Vol. 2, IEEE, 1996, pp. 966-971.

[27] M. Tsytsarau, T. Palpanas, Survey on mining subjective data on the web, Data Mining and Knowledge Discovery 24 (3) (2011) 478-514.

[28] K. Umamaheswari, V. Priya, Aspect Ranking Based on Author Specific Information Aggregation The Proposed Methodology, Journal of Scientific \& Industrial Research 75 (2016) 534-539.

[29] O. Vechtomova, Facet-based opinion retrieval from blogs, Information Processing and Management 46 (1) (2010) 71-88.

[30] D. Wang, S. Zhu, T. Li, SumView: A Web-based engine for summarizing product reviews and customer opinions, Expert Systems with Applications 40 (1) (2013) 27-33.

[31] K. Wang, J. N. Liu, W.-M. Ma, Towards the Detection of Potential Contradictions in Fuzzy Ontology Using a High Level Net Approach Integrated with Uncertainty Inference, in: 2010 IEEE International Conference on Data Mining Workshops, IEEE, 2010, pp. 883-890.

[32] R. R. Yager, On ordered weighted averaging aggregation operators in multicriteria decision making, IEEE transactions on Systems, Man and Cybernetics 18 (1) (1988) 183-190. 
[33] R. R. Yager, Quantifier guided aggregation using OWA operators, International Journal of Intelligent Systems 11 (1) (1996) 49-73.

[34] C. Zhang, D. Zeng, J. Li, F.-Y. Wang, W. Zuo, Sentiment analysis of Chinese documents: From sentence to document level, Journal of the American Society for Information Science and Technology 60 (12) (2009) 2474-2487.

[35] Z. Zhang, Weighing Stars: Aggregating Online Product Reviews for Intelligent E-commerce Applications, IEEE Intelligent Systems 23 (5) (2008) 42-49. 